\title{
High proliferation is associated with inferior outcome in male breast cancer patients
}

\author{
Cecilia Nilsson ${ }^{1,2}$, Anthoula Koliadi ${ }^{2}$, Ida Johansson ${ }^{3}$, Cecilia Ahlin ${ }^{4}$, Sten Thorstenson ${ }^{5}$, \\ Leif Bergkvist ${ }^{1}$, Ingrid Hedenfalk ${ }^{3}$ and Marie-Louise Fjällskog ${ }^{2}$ \\ ${ }^{1}$ Department of Oncology, Center for Clinical Research, Västmanland County Hospital, Västerås, Sweden; \\ ${ }^{2}$ Department of Radiology, Oncology and Radiation Science, University of Uppsala, Uppsala, Sweden; \\ ${ }^{3}$ Department of Oncology, Clinical Sciences and CREATE Health Strategic Center for Translational Cancer \\ Research, Lund University, Lund, Sweden; ${ }^{4}$ Department of Oncology, Örebro University Hospital, Örebro, \\ Sweden and ${ }^{5}$ Department of Pathology, Linköping University Hospital, Linköping, Sweden
}

\begin{abstract}
Assessment of proliferation is important in female breast cancer and individual treatment decisions are based upon its results, especially in the luminal subgroups. Gene expression analyses fail to group male breast cancer into the intrinsic subgroups previously established in female breast cancer. Even though proliferation has been shown to divide male breast cancer into molecular subgroups with different prognoses, the clinical importance of proliferation markers has not yet been elucidated. Previous studies in male breast cancer have demonstrated contradictory results regarding the prognostic impact of histological grade and Ki-67, parameters strongly associated with proliferation. The aim of the present project was to study proliferation in male breast cancer by assessing other proliferation-related markers viz. cyclins A, B, D1 and mitotic count. A total of 197 male breast cancer cases with accessible paraffin-embedded material and outcome data were investigated. Immunohistochemical stainings were performed on tissue microarrays. Kaplan-Meier estimates and the Cox proportional regression models were used for survival analyses with breast cancer death as the event. The subset of patients with high expression of cyclin A (hazard ratio (HR) $3.7 ; P=0.001$ ) and B (HR $2.7 ; P=0.02$ ) demonstrated a poorer survival. Furthermore, high mitotic count was associated with an increased risk of breast cancer death (HR 2.5; $\boldsymbol{P}=0.01$ ). In contrast, cyclin D1 overexpression was predictive of better breast cancer survival (HR 0.3; $\boldsymbol{P}=\mathbf{0 . 0 0 1 )}$. In conclusion, high levels of cyclin A and $B$ expression and an elevated mitotic count result in a two to threefold higher risk for breast cancer death, whereas cyclin D1 overexpression halves the risk. The clinical utility of these proliferation markers needs further elucidation.
\end{abstract}

Modern Pathology (2013) 26, 87-94; doi:10.1038/modpathol.2012.145; published online 24 August 2012

Keywords: breast cancer; immunohistochemistry; male

Male breast cancer represents $0.6 \%$ of all breast cancer and because of its rarity, specific treatment guidelines are lacking. Thus, male patients are treated according to the guidelines for female breast cancer. Breast cancer mortality among women has declined during the last decades because of improvements in diagnostic procedures and treatment, but the corresponding observation has not been made in male breast cancer. ${ }^{1}$ Recent studies have indicated that males have a poorer survival than females, despite having received adjuvant treatment

Correspondence: C Nilsson, PhD, MD, Department of Oncology, Center for Clinical Research, Västmanland County Hospital, Västerås SE-789 21, Sweden.

E-mail: cecilia.nilsson@ltv.se or cessan.nilsson@telia.com

Received 15 April 2012; revised 31 May 2012; accepted 31 May 2012; published online 24 August 2012 to the same extent. ${ }^{2,3}$ In addition, studies on transcriptional and genomic levels have revealed important molecular differences between male and female breast cancer. In a recent study, Johansson et $a l^{4}$ performed gene expression analyses in male breast cancer and concluded that male breast cancer tumors could not be classified into the intrinsic subtypes previously established in female breast cancer. In line with these data, comparative genomic hybridization demonstrated significant differences regarding DNA aberrations compared with female breast cancer, and a new subgroup unique for male patients was identified. ${ }^{5}$ Taking all these factors into account, these findings indicate that male breast cancer might be a separate tumor entity from female breast cancer.

Assessment of proliferation is important in female breast cancer and individual treatment decisions are 
based upon its results, especially in the luminal, hormone receptor positive subgroups. The clinical utility of proliferation in male breast cancer has not yet been fully elucidated. However, it is reasonable to assume that proliferation, being one of the hallmarks of cancer, is also of importance in male breast cancer.

Previous studies regarding immunohistochemical assessment of proliferation markers and tumor grade in male breast cancer are contradictory. ${ }^{6-9}$ In previous work from our group, a high percentage of tumors was considered as high proliferative as regards Ki-67 and Nottingham histological grade, but these variables did not provide any prognostic information (unpublished data). Nevertheless, in two recent studies investigating male breast cancer tumors on the transcriptional and genomic levels, proliferation was one of the most important key biological processes separating the male subgroups. ${ }^{4,5}$

Proliferation is driven by the cell cycle, which, in turn, is controlled by rapid (cyclic) changes in the levels of proteins called cyclin. Cyclins A and B are involved in the S- and/or M-phase. Cyclin D1 seems to have a dual role, being involved in the transition from G1 to S-phase, but also acts as a co-factor for the estrogen receptor (ER) $\alpha$. Ki-67 is a nuclear antigen present during all phases of the cell cycle. ${ }^{10}$

Several studies in female breast cancer have demonstrated that overexpression of Ki-67, cyclin $\mathrm{A}$ and cyclin B is associated with inferior outcome. ${ }^{11-15}$ Furthermore, results indicate that cyclins $A$ and B could have an increased prognostic impact when compared with Ki-67. ${ }^{12,15}$ The prognostic role of cyclin D1 in female breast cancer is unclear but some studies indicate that cyclin D1 may have a predictive role, with overexpression linked to tamoxifen resistance. ${ }^{16,17}$ Cyclin D1 has been evaluated in a few smaller studies of male breast cancer and overexpression seems to be associated with a better outcome. ${ }^{8,18}$ To date, the other cyclins have not been evaluated in male breast cancer.

Findings on the molecular level have shown that proliferation is of importance, but the clinical utility of proliferation markers in male breast cancer has not been confirmed. Male breast cancer might not be exactly the same disease as female breast cancer and it is important to know how to best assess proliferation and, if possible, to find out in which subset of patients information on proliferation is most valuable. The aim of this study was to investigate the prognostic impact of proliferation-related markers in male breast cancer.

\section{Materials and methods}

\section{Patients}

The National Cancer Register was used to identify male patients with invasive breast cancer diagnosed between 1990 and 2007 in two regions of Sweden
(Lund and Uppsala-Örebro), covering a total population of 3.66 million people. Only patients with available paraffin-embedded tumor blocks, clinicalpathological data and outcome data were included in the study, resulting in 109 patients from UppsalaÖrebro and 88 patients from Lund. Updated information on the patients' vital status and cause of death was retrieved from the National Population Register. The study was approved by the local ethical committee in Uppsala, Sweden.

\section{Tissue Microarray Construction}

Representative areas from each tumor block were punched and brought into recipient paraffin blocks to construct tissue microarrays. Two 1-mm cores from each tumor were transferred to the TMA block. From array blocks, $4 \mu \mathrm{m}$ thick sections were cut and transferred to glass slides.

\section{Immunohistochemistry}

The tissue microarray slides were deparaffinized in xylene and rehydrated through a ladder of graded ethanol (absolute ethanol, 95\%, 80\% and distilled water). For cyclin A (NCL-Cyclin A; NovoCastra Laboratories) and cyclin D1 (RM-9104-S; NeoMarkers) antigen retrieval was performed in a microwave oven for $10 \mathrm{~min}(750 \mathrm{~W})+15 \mathrm{~min}(350 \mathrm{~W})$ using a TE-buffer. For Cyclin B (1495-1; Epitomics) antigen retrieval was done in a pressure cooker for four minutes with the use of TRS buffer. After antigen retrieval, all tissue microarray slides were processed in an automatic immunohistochemistry staining machine according to the standard procedures (Autostainer, Dako, Sweden).

\section{Evaluation of Immunoreactivity Scores}

Cyclins A and D1 were analyzed by one investigator (CN) and cyclin B by a second investigator (AK). The percentage of positively stained cells was assessed by choosing the high-power field with the largest number of positively stained cells out of the two biopsies and dividing by the entire number of cells from the same high-power field. A minimum of 200 cells per tumor were counted. For cyclins A and D1, cells with unequivocal nuclear staining and for cyclin B, nuclear and cytoplasmic staining was considered as positive (See Figure 1 for example of cyclin A staining). Cells were manually counted in high-power fields using a light microscope. It has previously been shown that the reproducibility of cyclin scoring on tissue microarrays is good. ${ }^{15,19}$

Staining procedures and scoring of ER, PR, Ki-67, HER2 and Nottingham histological grade have been performed previously (unpublished data). Nottingham histological grade is composed of three different features-tubuli, nuclear atypia and mitotic 

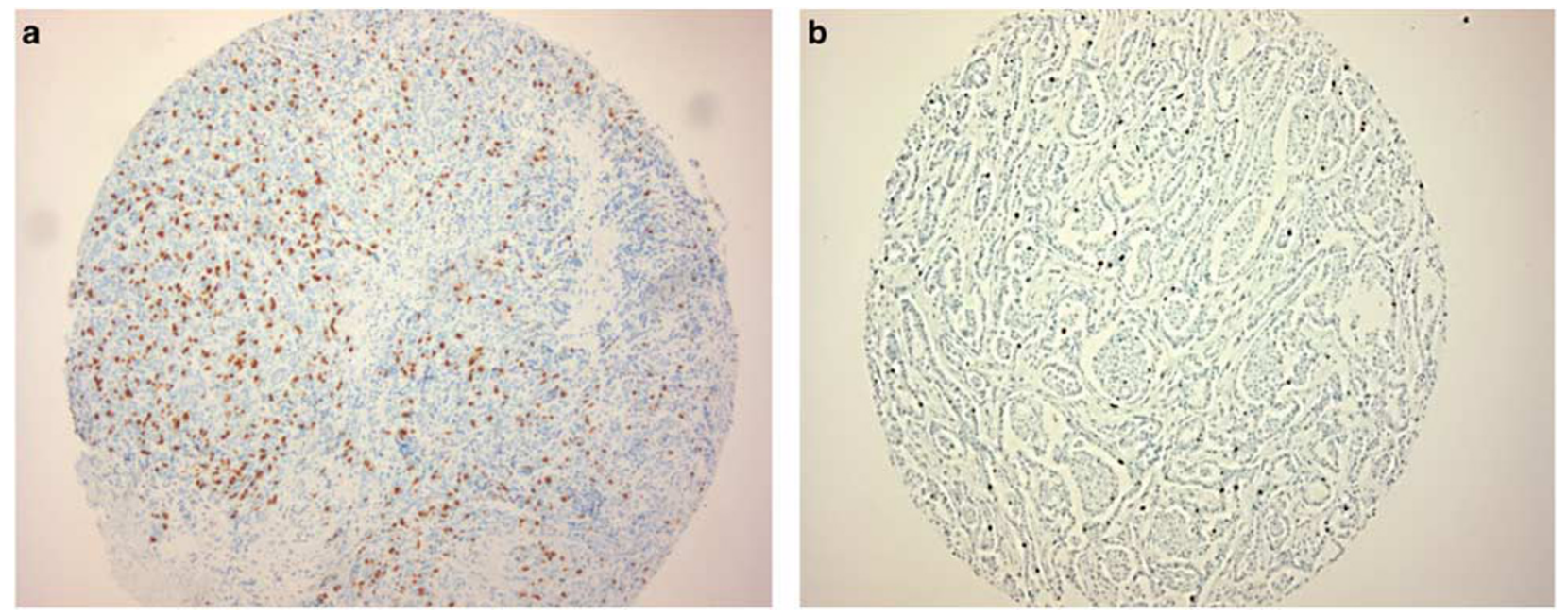

Figure 1 Cyclin A stainings in male breast cancer tumors. The first picture (a) is an example of a tumor with high expression and the second picture (b) illustrates a low expression.

count-which were analyzed as separate variables in the present study. The assessment of Nottingham histological grade was performed by a board-certified breast pathologist (ST) using a Zeiss Axioscope $\times 40$ objective (and $\times 10$ ocular) with a $0.43-\mathrm{mm}$ field of view.

\section{Evaluation of Proliferation Markers}

In the analyses of cyclins A and B and mitotic count, we used cutoffs previously defined in female breast cancer; cyclin A, $11 \%$; cyclin B, $7 \%$; and mitotic count, $>10$ per field of view. ${ }^{15,20}$ However, the cutoffs may not be the same in male breast cancer and, in order to find the optimal cutoffs for cyclin A, cyclin B and Ki-67, we used a previously described method. ${ }^{20}$ Briefly, the material was divided into 10 equal parts, so-called deciles, and the cutoff values corresponding to each decile limit were used to separate the material into a higher and a lower proliferating group. For each cutoff, the hazard ratio (HR) for breast cancer death was calculated, using Cox proportional hazard model. In previous studies on female breast cancer patients, the optimal cutoffs were found to correspond to the 7 th decile. ${ }^{15,20}$

\section{Statistics}

Correlations between variables were analyzed by Spearman's correlation test. The association between disease stage and treatment was tested using $\chi^{2}$ test. Cox proportional regression was used for uni- and multivariate analyses. Survival estimates were calculated with Kaplan-Meier and log-rank statistics. Breast cancer death was chosen as the end-point, other cases were censored. All $P$-values are two-sided with statistical significance set at 0.05 . For evaluation of cyclins in comparison with other clinicopathological variables, we used the results from a previous study on the same patient material (unpublished data). The SPSS software package (version 19) was used for all statistical analyses.

\section{Results}

\section{Patient Characteristics}

For clinicopathological and treatment characteristics, see Tables 1-2. A majority of patients had locoregional disease at diagnosis and underwent surgical, radiotherapeutic and systemic oncological treatment according to institutional guidelines. The mean follow-up time was 54 months (range 0-180). At the time of data collection, 41 of $197(21 \%)$ patients had died of breast cancer, and $82(42 \%)$ of other causes.

\section{Correlations}

Cyclins A and B, mitotic count and Ki-67 demonstrated strong positive correlation with each other. Cyclin A, but not cyclin B, showed an inverse correlation to PR, but none of these cyclins demonstrated any correlation with ER. Cyclin D1 was correlated to ER, but did not show any correlation with the other cyclins, Ki-67 or mitotic count (Table 3).

\section{The Optimal Cutoffs}

The mean values of expression for cyclin A, cyclin B and Ki-67 were as follows: cyclin A, 10\% (range 1$42 \%$ ); cyclin B, 9\% (range 0-29\%); and Ki-67, 16\% (range $0-83 \%$ ). The cutoff values for the 5th (median) and 7th decile were 9 and $12 \%$ for cyclin A, 8 and 11\% for cyclin B, and 14 and $21 \%$ for Ki- 
Table 3 Spearman's correlation test

\begin{tabular}{|c|c|c|c|c|c|c|c|c|}
\hline & Cyclin A & Cyclin B & Cyclin D1 & Ki67 & Mitosis & $E R$ & $P R$ & Tumor size \\
\hline Cyclin A & & $0.78^{\mathrm{a}}$ & 0.05 & $0.44^{\mathrm{a}}$ & $0.53^{\mathrm{a}}$ & -0.01 & $-0.19^{\mathrm{a}}$ & 0.11 \\
\hline Cyclin B & $0.78^{\mathrm{a}}$ & & 0.08 & $0.53^{\mathrm{a}}$ & $0.55^{\mathrm{a}}$ & 0.05 & -0.10 & $0.17^{\mathrm{a}}$ \\
\hline Cyclin D1 & 0.05 & 0.08 & & 0.06 & 0.07 & $0.24^{\mathrm{a}}$ & 0.05 & -0.12 \\
\hline Ki67 & $0.44^{\mathrm{a}}$ & $0.53^{\mathrm{a}}$ & 0.06 & & $0.32^{\mathrm{a}}$ & 0.08 & -0.06 & 0.11 \\
\hline Mitosis & $\mathbf{0 . 5 3}^{\mathrm{a}}$ & $0.55^{\mathrm{a}}$ & 0.07 & $0.32^{\mathrm{a}}$ & & 0.03 & -0.11 & $0.21^{b}$ \\
\hline ER & -0.01 & 0.05 & $0.24^{\mathrm{a}}$ & 0.08 & 0.03 & & $0.28^{\mathrm{a}}$ & -0.10 \\
\hline $\mathrm{PR}$ & $-0.19^{\mathrm{a}}$ & -0.10 & 0.05 & -0.06 & -0.11 & $0.28^{\mathrm{a}}$ & & -0.09 \\
\hline Tumor size & 0.11 & $0.17^{\mathrm{a}}$ & -0.12 & 0.11 & $0.21^{\mathrm{a}}$ & -0.10 & -0.09 & \\
\hline
\end{tabular}

ER, estrogen receptor.

asignificant correlation.

bstrong correlations $(>0.4)$ are marked in bold.

in any notable change of the HR for endocrine treatment; 2.34 (95\% CI, 1.02-5.37) vs 2.14 (95\% CI, $0.88-5.19$ ) in the unadjusted and adjusted analyses, respectively.

\section{Discussion}

In the present study, we found that overexpression of cyclin A and cyclin B, and a high mitotic count were predictive of poorer breast cancer survival in male breast cancer. Ki-67 was re-evaluated using different cutoffs but did not demonstrate any prognostic value. Contrary to the other cyclins, overexpression of Cyclin D1 was an independent predictor of a better breast cancer survival.

The median values of cyclins A, B and Ki-67 were in accordance with previous female breast cancer materials, indicating that there are no major differences in the prevalence of the proliferation marker expression between male and female breast cancer. ${ }^{15,20}$ Cyclin A, cyclin B, mitotic count and Ki-67 were strongly positively correlated to each other, verifying that these variables are linked in the tumor's biological background. When using cutoffs previously defined in female breast cancer, cyclin A overexpression showed a tendency to be associated with an increased risk for breast cancer death. However, the optimal cutoffs for cyclins A or B may be different in male breast cancer, and, in order to define the best cutoff, a previously described method was used. ${ }^{20}$ We found that overexpression of cyclins A and B was associated with poorer outcome when using the cutoffs corresponding to the subset of tumors with the highest expression. In addition, we could demonstrate that a high mitotic count was associated with an increased risk of breast cancer death, which further strengthens the conclusion that proliferation is of prognostic importance in male breast cancer. Ki-67 did not demonstrate any prognostic value in this cohort, but some studies in female breast cancer have shown that Ki67 may be a weaker prognostic factor compared with cyclins $\mathrm{A}$ and $\mathrm{B}^{12,15}$ and, hence, a larger cohort may be needed to be able to establish its prognostic
Table 4 Prognostic impact of proliferation markers in comparison with other clinicopathological parameters

\begin{tabular}{|c|c|c|c|}
\hline & \multicolumn{3}{|c|}{ Univariate models } \\
\hline & $H R$ & $95 \% C I$ & $\mathrm{P}$ \\
\hline \multicolumn{4}{|l|}{ Nodal status } \\
\hline pNo (ref.) & 1.0 & & \\
\hline pN1 & 5.4 & $2.2-13.0$ & $<0.01$ \\
\hline \multicolumn{4}{|l|}{ Tumor size } \\
\hline$\leq 20 \mathrm{~mm}$ (ref.) & 1.0 & & \\
\hline$>20 \mathrm{~mm}$ & 2.9 & $1.5-5.7$ & $<0.01$ \\
\hline \multicolumn{4}{|l|}{$E R$} \\
\hline$>10 \%$ (ref.) & 1.0 & & \\
\hline$\leq 10 \%$ & 6.2 & $2.7-14.2$ & $<0.01$ \\
\hline \multicolumn{4}{|l|}{$P R$} \\
\hline$>10 \%$ (ref.) & 1.0 & & \\
\hline$\leq 10 \%$ & 2.4 & $1.2-4.7$ & 0.01 \\
\hline \multicolumn{4}{|l|}{ Cyclin A } \\
\hline$\leq 17 \%$ (ref.) & 1.0 & & \\
\hline$>17 \%$ & 3.7 & $1.6-8.6$ & $<0.01$ \\
\hline \multicolumn{4}{|l|}{ Cyclin B } \\
\hline$\leq 15 \%$ (ref.) & 1.0 & & \\
\hline$>15 \%$ & 2.7 & $1.2-6.2$ & 0.02 \\
\hline \multicolumn{4}{|l|}{ Cyclin D1 } \\
\hline$\leq 62 \%$ (ref.) & 1.0 & & \\
\hline$>62 \%$ & 0.3 & $0.2-0.7$ & $<0.01$ \\
\hline \multicolumn{4}{|l|}{ Mitotic count } \\
\hline$\leq 10$ (ref.) & 1.0 & & \\
\hline$>10$ & 2.5 & $1.3-5.0$ & 0.01 \\
\hline
\end{tabular}

Univariate analyses using Cox proportional hazard regression model.

impact in male breast cancer. Another issue with potential influence on our results is that a subset of our patients had received adjuvant and/or palliative chemotherapy treatment, which is known to diminish the prognostic impact of proliferation markers as proliferation is predictive of chemotherapy response. ${ }^{20,21}$ This is exemplified in a study investigating cyclin A and Ki-67 in female breast cancer, in which the overexpression of cyclin A and Ki-67 was predictive of inferior breast cancer survival only in 

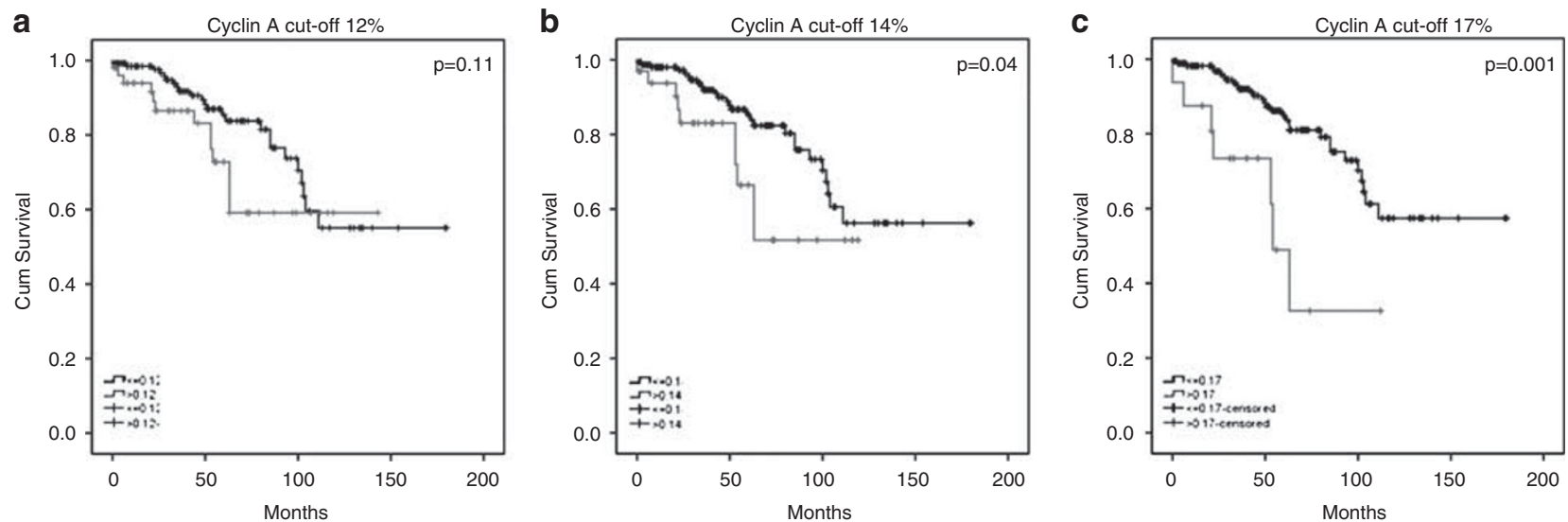

Figure 2 Kaplan-Meier survival analyses of cyclin A using three different cutoffs. The black line represents lower expression and the gray line higher expression. (a) Cutoff $12 \%$, corresponding to the 7 th decile $(P=0.11)$. (b) Cutoff $14 \%$, corresponding to the 8 th decile $(P=0.04)$. (c) Cutoff $17 \%$, corresponding to the 9th decile $(P=0.001)$.

chemo-naive patients. ${ }^{20}$ Similarly, in our study, mitotic count was highly predictive of breast cancer death in the chemo-naïve subgroup (HR 6.4, 95\% CI 1.9-21.3, $P=0.003)$ compared with the chemo-treated subgroup (HR 1.9, 95\% CI $0.3-11.9$, $P=0.47)$. There were too few events to allow for the corresponding analyses of the other proliferation markers. This stratification was not preplanned and underpowered why it should be interpreted with caution. Some previous studies in male breast cancer have indicated a poorer outcome among patients whose tumors were considered highly proliferating by immunohistochemical assessment or tumor grade, which is in line with our results, ${ }^{6,8,22}$ although other studies have not been able to confirm this observation. ${ }^{7,9}$

Two recent studies on the molecular level have shown that proliferation is important in the distinction between high- and low-risk male breast cancer tumors. In a paper characterizing male breast cancer tumors by comparative genomic hybridization, two distinct subgroups could be identified, which differed from genomic subgroups of female breast cancer. ${ }^{5}$ Tumors in the larger male breast cancer subgroup displayed complex genomic profiles, significantly higher proliferation and were associated with a poor outcome. In the other study, characterizing male breast cancer tumors by gene expression, two male breast cancer subgroups largely corresponding to the comparative genomic hybridizationbased groups were identified. The male subgroups did not resemble any of the widely acknowledged intrinsic subgroups of female breast cancer. The larger male subgroup, encompassing $70 \%$ of patients, displayed higher proliferation scores in addition to other characteristics, indicating a more aggressive tumor behavior. ${ }^{4}$ In both studies, the patient cohorts were small owing to the rareness of the disease and the authors stated that male breast cancer subgroups may be further refined, but larger cohorts are needed. However, the results clearly show that male breast cancer is a heterogeneous disease and that the male breast cancer subgroups are different from female breast cancer subgroups.

This is in line with the notion that molecular subtypes assessed by immunohistochemistry using classifications validated in female breast cancer are not directly transferable to male breast cancer (unpublished data). How to best define male breast cancer subgroups by immunohistochemical markers has yet to be determined, but as shown in the present study, proliferation is a biological process of importance in this context.

Cyclin D1 did not correlate to the other cyclins, Ki-67 or mitotic count in our material, suggesting that cyclin D1 is involved in tumor biological processes other than proliferation. This is in accordance with the reports in female breast cancer showing that overexpression of cyclin D1 does not correlate to the other cyclins and is more often seen in ER positive and well-differentiated tumors. ${ }^{23}$ Studies evaluating the prognostic role of cyclin D1 overexpression in female breast cancer are contradictory, with some reporting a better ${ }^{23-25}$ and others a poorer outcome. ${ }^{16,26}$ We could demonstrate that overexpression of cyclin D1 is predictive of better breast cancer survival in male breast cancer, which has been indicated in previous reports in male breast cancer. ${ }^{8,18}$ The reason for cyclin D1 overexpression seeming to be associated with a better outcome despite its proliferation-activating potential is not fully understood. One hypothesis is that there might be a complex interaction between key biological processes in the development of metastases. An in vitro study demonstrated that a decreased expression of cyclin D1 was linked to an enhanced infiltrative potential in the tumor, explained by the finding that cells in the quiescent phase had a greater migratory capacity. ${ }^{27}$ The authors of the latter study strengthened their finding by demonstrating that cyclin D1 expression was inversely associated with tumor size in a female breast cancer material. This observation could not be verified in our material. However, on the transcriptional level, a 
majority of male breast cancer tumors were strongly associated with gene-expression patterns representing tumor migration, which supports the hypothesis that this is a key tumor biological process in male breast cancer. ${ }^{4}$

Cyclin D1 overexpression has been linked to tamoxifen-resistance in FBC. ${ }^{16,17}$ It has been suggested that cyclin D1 has the ability to stimulate ER $\alpha$ in a ligand-independent manner, ${ }^{28,29}$ and could as such reverse the ER $\alpha$-blocking effect of tamoxifen. This could be of importance in male breast cancer, where tamoxifen is considered to be the golden standard in adjuvant treatment, although there are no prospective studies that confirm its efficacy. There are no studies addressing the issue of tamoxifen resistance in male breast cancer. In our study, patients undergoing adjuvant endocrine treatment had a poorer breast cancer survival compared with cases without such treatment. The most plausible explanation for this finding is that treatment was given more often in higher-stage disease. However, the prognostic impact of adjuvant endocrine treatment did not change when adjusting for cyclin D1, indicating that cyclin D1 does not influence the response to endocrine treatment in male breast cancer. One reason for this could be that ER signaling is different in MBC, which was suggested in a study where a subgroup of male breast cancers displayed a diminished ER signature, even though a vast majority of tumors were ER positive by immunohistochemistry. In contrast, a decreased ER signature was only observed in ER negative female breast cancer tumors. ${ }^{4}$ Other studies have also indicated a different role of steroid receptors in male breast cancer, suggesting a reduced functionality of ER. ${ }^{30,31}$ Consequently, the ER stimulating effect of cyclin D1 may be limited in male breast cancer.

In summary, cyclin D1 expression is a positive prognostic marker in male breast cancer, although its predictive value needs to be further investigated.

Although this is one of the largest series of male breast cancer with available tumor tissue and outcome data, the moderate power makes interpretation of nonsignificant results difficult, and hence requires validation. Nevertheless, in addition to the reliable information on vital status and death cause, access to clinicopathological and treatment data strengthen the study. Furthermore, the patients were identified from a population-based register with near-complete coverage, leading to an unbiased patient selection.

In conclusion, our observations show that proliferation is important in male breast cancer and that cyclins and mitotic count may provide more reliable prognostic information compared with Ki-67, which is today widely used when assessing breast cancer prognosis. Cyclin D1 adds independent prognostic information; however, its predictive value is still unclear. Further studies are warranted to establish the optimal marker and cutoff values.

\section{Acknowledgements}

We thank all the involved Pathology Departments for providing tissue material for the study. This study was supported by grants from the Regional Research Foundation in Uppsala-Örebro, the Lion's Cancer Foundation, University Hospital, Uppsala and Västmanlands Research Foundation. We acknowledge the Associate Professor Henry Letocha for linguistic support.

\section{Disclosure/conflict of interest}

The authors declare no conflict of interest.

\section{References}

1 Miao H, Verkooijen HM, Chia KS, et al. Incidence and outcome of male breast cancer: an international population-based study. J Clin Oncol 2011;29:43814388.

2 Czene K, Bergqvist J, Hall P, et al. How to treat male breast cancer. Breast 2007;16(Suppl 2):S147-S154.

3 Nilsson C, Holmqvist M, Bergkvist L, et al. Similarities and differences in the characteristics and primary treatment of breast cancer in men and women - a population based study (Sweden). Acta Oncol 2011;50: 1083-1088.

4 Johansson I, Nilsson C, Berglund P, et al. Gene expression profiling of primary male breast cancers reveals two unique subgroups and identifies N-acetyltransferase-1 (NAT1) as a novel prognostic biomarker. Breast Cancer Res 2012;14:R31.

5 Johansson I, Nilsson C, Berglund P, et al. Highresolution genomic profiling of male breast cancer reveals differences hidden behind the similarities with female breast cancer. Breast Cancer Res Treat 2011;129: 747-760.

6 Cutuli B, Le-Nir CC, Serin D, et al. Male breast cancer. Evolution of treatment and prognostic factors. Analysis of 489 cases. Crit Rev Oncol Hematol 2010;73:246-254.

7 Giordano SH, Cohen DS, Buzdar AU, et al. Breast carcinoma in men: a population-based study. Cancer 2004;101:51-57.

8 Rayson D, Erlichman C, Suman VJ, et al. Molecular markers in male breast carcinoma. Cancer 1998;83: 1947-1955.

9 Wang-Rodriguez J, Cross K, Gallagher S, et al. Male breast carcinoma: correlation of ER, PR, Ki-67, Her2Neu, and p53 with treatment and survival, a study of 65 cases. Mod Pathol 2002;15:853-861.

10 Colozza M, Azambuja E, Cardoso F, et al. Proliferative markers as prognostic and predictive tools in early breast cancer: where are we now? Ann Oncol 2005;16: 1723-1739.

11 Agarwal R, Gonzalez-Angulo AM, Myhre S, et al. Integrative analysis of cyclin protein levels identifies cyclin b1 as a classifier and predictor of outcomes in breast cancer. Clin Cancer Res 2009;15:3654-3662.

12 Ahlin C, Zhou W, Holmqvist M, et al. Cyclin A is a proliferative marker with good prognostic value in node-negative breast cancer. Cancer Epidemiol Biomarkers Prev 2009;18:2501-2506. 
13 Luporsi E, Andre F, Spyratos F, et al. Ki-67: level of evidence and methodological considerations for its role in the clinical management of breast cancer: analytical and critical review. Breast Cancer Res Treat 2011;132:895-915.

14 Michalides $\mathrm{R}$, van Tinteren $\mathrm{H}$, Balkenende $\mathrm{A}$, et al. Cyclin A is a prognostic indicator in early stage breast cancer with and without tamoxifen treatment. Br J Cancer 2002;86:402-408.

15 Nimeus-Malmstrom E, Koliadi A, Ahlin C, et al. Cyclin B1 is a prognostic proliferation marker with a high reproducibility in a population-based lymph node negative breast cancer cohort. Int J Cancer 2010;127: 961-967.

16 Rudas M, Lehnert M, Huynh A, et al. Cyclin D1 expression in breast cancer patients receiving adjuvant tamoxifen-based therapy. Clin Cancer Res 2008;14: 1767-1774.

17 Stendahl M, Kronblad A, Ryden L, et al. Cyclin D1 overexpression is a negative predictive factor for tamoxifen response in postmenopausal breast cancer patients. Br J Cancer 2004;90:1942-1948.

18 Kanthan R, Fried I, Rueckl T, et al. Expression of cell cycle proteins in male breast carcinoma. World J Surg Oncol 2010;8:10.

19 Aaltonen K, Ahlin C, Amini RM, et al. Reliability of cyclin A assessment on tissue microarrays in breast cancer compared to conventional histological slides. Br J Cancer 2006;94:1697-1702.

20 Ahlin C, Aaltonen K, Amini RM, et al. Ki67 and cyclin A as prognostic factors in early breast cancer. What are the optimal cut-off values? Histopathology 2007;51: 491-498.

21 Miglietta L, Vanella P, Canobbio L, et al. Prognostic value of estrogen receptor and Ki-67 index after neoadjuvant chemotherapy in locally advanced breast cancer expressing high levels of proliferation at diagnosis. Oncology 2010;79:255-261.
22 Pich A, Margaria E, Chiusa L. Proliferative activity is a significant prognostic factor in male breast carcinoma. Am J Pathol 1994;145:481-489.

23 Bostrom P, Soderstrom M, Palokangas T, et al. Analysis of cyclins A, B1, D1 and E in breast cancer in relation to tumour grade and other prognostic factors. BMC Res Notes 2009;2:140.

24 Hwang TS, Han HS, Hong YC, et al. Prognostic value of combined analysis of cyclin D1 and estrogen receptor status in breast cancer patients. Pathol Int 2003;53: 74-80.

25 Lundgren K, Brown M, Pineda S, et al. Effects of cyclin D1 gene amplification and protein expression on time to recurrence in postmenopausal breast cancer patients treated with anastrozole or tamoxifen: a TransATAC study. Breast Cancer Res 2012;14:R57.

26 Aaltonen K, Amini RM, Landberg G, et al. Cyclin D1 expression is associated with poor prognostic features in estrogen receptor positive breast cancer. Breast Cancer Res Treat 2009;113:75-82.

27 Lehn S, Tobin NP, Berglund P, et al. Down-regulation of the oncogene cyclin D1 increases migratory capacity in breast cancer and is linked to unfavorable prognostic features. Am J Pathol 2010;177:2886-2897.

28 Lamb J, Ladha MH, McMahon C, et al. Regulation of the functional interaction between cyclin D1 and the estrogen receptor. Mol Cell Biol 2000;20:8667-8675.

29 Neuman E, Ladha MH, Lin N, et al. Cyclin D1 stimulation of estrogen receptor transcriptional activity independent of cdk4. Mol Cell Biol 1997;17:5338-5347.

30 Callari M, Cappelletti V, De Cecco L, et al. Gene expression analysis reveals a different transcriptomic landscape in female and male breast cancer. Breast Cancer Res Treat 2010;127:601-610.

31 Weber-Chappuis K, Bieri-Burger S, Hurlimann J. Comparison of prognostic markers detected by immunohistochemistry in male and female breast carcinomas. Eur J Cancer 1996;32A:1686-1692. 